\title{
TV/Series
}

$2 \mid 2012$

Les séries télévisées dans le monde : Échanges, déplacements et transpositions

\section{Promenades au cœur du Village : L'articulation des espaces dans la série Le Prisonnier}

\section{Valérie Foulquier}

\section{(2) OpenEdition \\ Journals}

Édition électronique

URL : http://journals.openedition.org/tvseries/1389

DOI : 10.4000/tvseries.1389

ISSN : 2266-0909

Éditeur

GRIC - Groupe de recherche Identités et Cultures

Référence électronique

Valérie Foulquier, « Promenades au cœur du Village : L'articulation des espaces dans la série Le

Prisonnier », TV/Series [En ligne], 2 | 2012, mis en ligne le 01 novembre 2012, consulté le 30 avril 2019 URL : http://journals.openedition.org/tvseries/1389; DOI : 10.4000/tvseries.1389

\section{(c) (i) (9)}

TV/Series est mis à disposition selon les termes de la licence Creative Commons Attribution - Pas d'Utilisation Commerciale - Pas de Modification 4.0 International. 


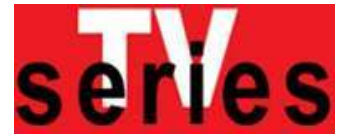

\title{
Promenades au cour du Village : L'articulation des espaces dans la série Le Prisonnier' \\ Valérie FOULQUIER
}

\begin{abstract}
L'intrigue du Prisonnier est à l'évidence une histoire d'enfermement et d'impossible évasion. Le héros est un espion qui vient de démissionner à grand fracas : le passeport, les clichés de plages tropicales qu'il jette dans sa valise, entrouvrent un espace de renouveau possible. Mais cet espace lui sera farouchement refusé ; il est enlevé puis déporté au Village, lieu clos et mystérieux d'où il n'aura de cesse d'essayer de s'échapper et dont la localisation demeurera mystérieuse. De par son indétermination toponymique et géographique, cet espace autarcique va se prêter à de nombreuses articulations narratologiques. Cet article se propose d'explorer le Village dans ses différentes strates et d'en dessiner le plan sous la double modalité de la perspective et du déplacement. Prédicat matériel d'une esthétique carcérale omniprésente, il n'est pas qu'un " mauvais lieu » (dys-topia), il est aussi un espace de séduction. Etymologiquement, il « emmène à part, à l'écart » (seducere : " mener hors du droit chemin ») et entraine les spectateurs dans un univers ludique et labyrinthique. Par des déplacements de perspective, il se fait lieu de récréation sensorielle et nous captive. Mais le Village opère un autre glissement : sa mise en scène du lieu et des figures glisse progressivement de l'espace organisé et rationnel pour nous faire pénétrer les espaces intimes de l'imagination et de l'inconscient. Ce déplacement est à l'origine d'une véritable poétique du décloisonnement, créant pour la série un univers onirique aux accents surréalistes.
\end{abstract}

$\mathrm{U}$

n bruit de tonnerre, des nuages - symboles du mouvement et de l'inconstance des apparences -, une route à perte de vue que vient fendre une Lotus Seven immatriculée KAR120C : les tout premiers plans du Prisonnier sont incontestablement traités sur le double mode du déplacement et de la perspective ${ }^{2}$. La ligne de fuite constituée par cette route anonyme, légèrement en plongée, ouvre déjà un espace infini, sans repère, vertigineux. Si le spectateur peut un instant se localiser (la Lotus longe Big Ben et le palais de Westminster - nous sommes donc à Londres), la caméra brouille les pistes vers de nouveaux lieux indéterminés, insaisissables : un parking souterrain, un tunnel sombre, un bureau secret. De l'échange houleux entre le héros et le bureaucrate, rien ne sera transcrit, la musique extra-diégétique et les bruits de tonnerre faisant barrière à toute élucidation concernant l'action et sa caractérisation spatiale. Nous comprenons que les monuments londoniens font dès lors moins référence à un lieu précis qu'à un imaginaire britannique particulier, empreint de mystère, d'espionnage (genre très en vogue dans les années 60) et d'une tradition orwellienne d'univers dystopiques. Patrick McGoohan, alors très connu pour son rôle d'agent secret dans la série Destination danger (Danger Man, ITV, 1960-66), est, on le devine, un espion qui

${ }^{1}$ The Prisoner, ATV Midlands, 1967-68, série de 17 épisodes créée par George Markstein et Patrick McGoohan.

${ }^{2}$ Le générique peut être visionné ici (lien consulté en août 2012)

www.youtube.com/watch?v=14eUKogPF7s 
vient de démissionner : le passeport, les clichés de plages tropicales qu'il jette dans sa valise entrouvrent un espace de renouveau possible. Mais cet espace lui sera farouchement refusé ; il est enlevé puis déporté. Où est-il ? Au Village, lieu clos et mystérieux d'où il n'aura de cesse d'essayer de s'échapper et dont la localisation demeurera impossible3. Le tout premier dialogue, dans l'épisode pilote « Arrival », est échangé entre le héros, désormais apparenté à un simple numéro, le 6, et l'aubergiste :

L'AUBERGISTE. We'll be opening in a minute.

NUMÉRO 6. What's the name of this place?

L'AUBERGISTE. You're new here, aren't you?

NUMÉRO 6. Where?

L'AUBERGISTE. Do you want breakfast?

NUMÉRO 6. Where is this?

L'AUBERGISTE. The Village.

Relevons aussi l'échange récurrent avec le Numéro 2, lors des génériques suivants :

NUMÉRO 6. Where am I?

NUMÉRO 2. In the Village [...]

NUMÉRO 6. Whose side are you on?

NUMÉRO 2. That would be telling.

L’accroche mystérieuse, nécessaire à ce stade pour séduire et captiver le spectateur, naît du lieu même et de son indétermination toponymique. Ce n'est pas la matérialité de l'espace qui semble primer ici mais bien plutôt l'allégorie, l'idée «Village ». Elle ne se laisse pas appréhender concrètement; ses contours demeurent évasifs comme l'atteste la carte de la région : à l'ouest, à l'est et au nord, ce ne sont que " Mountains », au sud, " The Sea » (épisode 1, « Arrival »)4.

Tautologiques, insituables, ces repères géographiques n'indiquent qu'une chose : que le Village est autre. Autre part, autre chose. C'est pourquoi cet article se propose d'explorer le traitement du Village dans la série et d'en dessiner le plan sous la double modalité de la perspective et du déplacement. Nous verrons comment ce village dystopique, après avoir exploité les différents ressorts de l'enfermement, transpose les spectateurs dans un lieu de récréation sensorielle fondée sur des jeux de perspective. Nous entamerons alors une lente promenade intérieure, au gré d'une syntaxe surréaliste, vers

3 Voir l'épisode « Many Happy Returns », ou épisode 7. L’ordre des épisodes étant au centre de controverses entre diffuseurs, créateurs et amateurs de la série, cet article mentionnera l'ordre d'apparition lors de la toute première diffusion à la télévision (1er octobre 1967-4 février 1968)

${ }^{4}$ Voir la réplique de la carte aperçue dans l'épisode 1, « Arrival ", consultable à l'adresse http://www.chronoludic.com/2011/02/i-live-in-the-village-so-why-cant-i-play-theprisoner/ (lien consulté en août 2012). 
un déploiement délirant de l'espace intime, vers une véritable poétique du décloisonnement.

Commençons par l'évidence : l'intrigue du Prisonnier est une histoire d'enfermement et d'impossible évasion dans laquelle tout participe de la thématique carcérale, en particulier le traitement de l'espace et du mouvement. Entité autarcique et autonome, le Village est une forteresse conçue contre un danger qui viendrait non pas de l'extérieur mais de l'intérieur : son rôle est de tout contenir et figer. En cela, le Village apparaît dans un premier temps comme le lieu de la clôture, négation du déplacement et de la perspective dans son acception d'horizon. C'est un microcosme à part entière, où de nombreuses langues sont employées et comprises par les différentes communautés ( «It's very cosmopolitan, you never know who you may meet» confie la jeune femme asiatique au volant du taxi, dans l'épisode 1, «Arrival »). Certaines sont difficilement identifiables : par exemple, dans ce même épisode, Numéro 6 intercepte la conversation de l'épicier, qui pourrait être de l'espéranto. L'allusion à cette langue au dessein universel ne vient que renforcer les dimensions cosmopolite et utopique d'un espace qui se désire cloisonné mais global : " The whole Earth as the Village ", comme le résume Numéro 6 dans l'épisode 2, « The Chimes of Big Ben ». Les clivages idéologiques Est/Ouest ne sont ici pas de mise car tout est simplement « international », même la cuisine ${ }^{5}$. Le Village possède sa propre monnaie (« unit»), ses propres codes de politesse («Be seeing you !») et, on le comprend vite, ses propres valeurs sociétales. Lors de la visite panoramique en hélicoptère offerte par le tout premier Numéro 2, celui-ci confirme notre sentiment :

Quite a beautiful place, isn't it? Almost like a world on its own. [...] We have everything here, water, electricity. [...] We have our own Council, democratically elected. [...] We also have our own graveyard.

Cette réplique semble rappeler la définition quelque peu ironique de la communauté autonome par Nathaniel Hawthorne dans The Scarlet Letter (1850) :

The founders of a new colony, whatever Utopia of human virtue and happiness they might originally project, have invariably recognised it among their earliest practical necessities to allot a portion of the virgin soil as a cemetery, and another portion as the site of a prison ${ }^{6}$.

Si le Village a son cimetière, penchons-nous à présent sur la prison. En suivant les déambulations de Numéro 6 , le spectateur se familiarise peu à peu avec ce décor paisible et sinueux. Mais, sous l'apparence d'un

5 Voir l'épisode « Free for All » (épisode 4).

${ }^{6}$ Nathaniel Hawthorne, The Scarlet Letter, New York, Penguin Books, 1983 [1850], chapitre 1, p. 75 
village souriant de villégiature, se dessine bientôt l'une des figures les plus connues de l'imaginaire carcéral - la structure panoptique décrite par Michel Foucault, à la suite de Jérémy Bentham, dans Surveiller et Punir7. Le panoptique est un bâtiment circulaire (que vient symboliser, dans Le Prisonnier, le Village tout entier), au centre duquel se situe la tour du Gardien (ici, le Dôme Vert, quartier stratégique du Numéro 2, dépositaire de l'attribut suprême : voir sans être vu). Le bâtiment périphérique du panoptique est divisé en cellules (les appartements du Village, paradoxalement appelés « Private ${ }^{8}$ »), traversées par une lumière extérieure persistante permettant une lisibilité totale de l'intimité des « résidents ». Ce viol de l'intime s'opère dans la série par le truchement de technologies de contrôle futuristes - pour l'époque -, commandées depuis le Dôme. Le détenu ne sait jamais s'il est regardé mais doit être sûr qu'il peut toujours l'être : c'est ce que nous rappellent les plans serrés récurrents sur le Dôme Vert. Dans Le Prisonnier, l'idée de tout voir est omniprésente. Avant de démissionner, Numéro 6 était un agent secret chargé de surveiller et de rapporter des informations. Il est désormais à son tour épié et étudié. Cette transposition opère une mise en abyme par laquelle se dédouble le statut des spectateurs, à la fois voyeurs et captifs d'un système vertigineux et fascinant.

Que voit-on dans ce village ? Des habitants aux mines enjouées et aux habits bariolés, en perpétuel mouvement. Ils marchent, déambulent, paradent ; s'ils discutent au coin d'une terrasse, ils n'y restent jamais vraiment longtemps et les échanges paraissent superficiels. Très peu d'informations nous sont fournies quant à leurs déplacements et occupations. Il semblerait que leur fonction première soit réellement d'imprégner le Village d'un flux constant de mouvement. Cette activité de surface fonctionnerait dès lors comme une épaisseur additionnelle du décor, façonnant un espace dynamique qui inviterait Numéro 6 - et les spectateurs - à emprunter un cheminement plus mental que spatial : si la caméra ne nous mène, précisément, nulle part, au moins pouvons-nous laisser déambuler notre esprit? Des détails viennent renforcer l'artificialité chorégraphique. C'est par exemple le cas du bateau immobile dans le port factice et sablonneux de La Pension. C'est aussi, et surtout, le symbole du village : un vélocipède dont les roues ne tournent pas (voir figure 1$)^{9}$.

\footnotetext{
7 Michel Foucault, Surveiller et Punir, Paris, Gallimard, Coll. Tel., 1975.

${ }^{8}$ La cellule de Numéro 6, ses gadgets « high-tech » et la salle de contrôle circulaire peuvent être visionnés ici (lien consulté en août 2012) :

http://www.youtube.com/watch?v=Xs1ekTX 8zM

${ }^{9}$ Il est intéressant de noter la tentative de recomposition systématique de ce mode de transport disloqué lors du générique de fin. Le générique de fin peut-être visionné ici (lien consulté en août 2012) :

http://www.youtube.com/watch?v=fQ9AqRAwgK4\&feature=related
} 


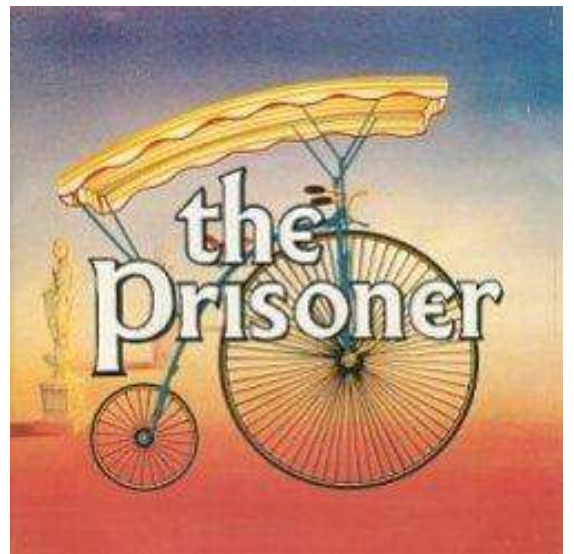

Fig. 1 : Le générique de fin

Comme pour renforcer cette vacuité des mouvements, les habitants reçoivent parfois l'injonction de s'immobiliser et de se taire. Anéantissant toute profondeur et perspective, ils se fondent dans le décor, pétrifiés dans le temps et l'espace: "they go grey ", pour reprendre une expression propre aux services secrets britanniques. $\mathrm{Au}-$ delà de la métaphore qui lui donne sens dans ce contexte (« passer inaperçu »), la notion littérale de gris est ici intéressante car paradoxale : tout est criard au Village, mais, comme on le comprend vite, il ne s'agit que de surface et derrière l'apparente gaieté irisée se cache une épaisseur plus sombre et plus étrange. Ce glissement du familier à l'étrange est sans conteste matérialisé par la figure inquiétante du Rôdeur (« Rover », voir figure 2).

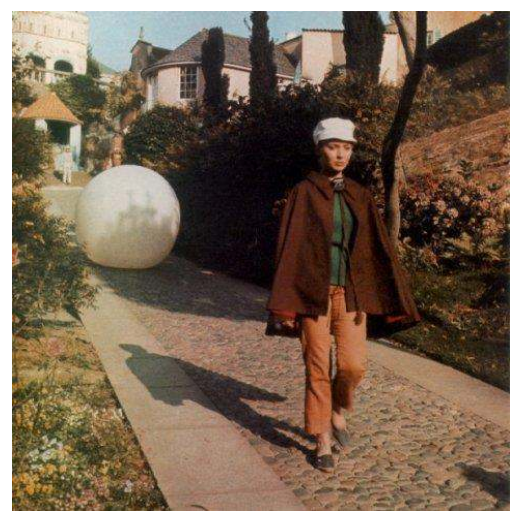

Fig. 2 : Le Rôdeur suivant une habitante dans l'épisode 8, « Dance of the Dead ». 
Issue des profondeurs marines, toujours associée à un cri primal et terrifiant, cette énorme boule blanche dotée d'une impulsion autonome de Cerbère a pour unique fonction d'empêcher les échanges et l'articulation des espaces. Le Rôdeur régule les déplacements et endigue le moindre tumulte. Il est, en ce sens, l'aboutissement cauchemardesque de l'univers carcéral délimité par le Village ${ }^{10}$.

Derrière une apparente liberté chorégraphique se dévoile bien vite un immobilisme totalitaire duquel les hommes ne semblent pourtant pas souffrir : les visages restent placides et souriants dans cette cage dorée et ludique. Tout semble question de perspective. C'est que l'enfermement n'est pas immédiatement visible : il est avant tout rhétorique. Comme cette thématique, profondément féconde de par ses implications intertextuelles (Victor Klemperer et la Lingua Tertii Imperii ou LTI, George Orwell et la Novlangue...) dépasse le cadre imparti par la présente étude, nous nous contenterons de relever quelques aphorismes du Village pour illustrer notre propos : «A still tongue makes a happy life », "Questions are a burden to others/ Answers are a prison for oneself »... Aucune question ne reçoit, en effet, de réponse dans cette série qui enferme le langage et les échanges dans un espace clos et stérile. L'esprit des résidents ne déambule plus, prisonnier d'une autorité qui assujettit et pétrifie. Pour eux, il n'y a nulle part où aller. Et pour nous ?

Le Village s'appréhende comme le prédicat matériel d'une esthétique carcérale, mais il dévoile peu à peu d'autres ressorts narratologiques. Il n'est pas que le «mauvais lieu » (dys-topia), il est aussi un espace de séduction. Étymologiquement, il « emmène à part, à l'écart » (seducere : «mener hors du droit chemin ») ; et c'est bien ce fonctionnement du Village qui nous intéresse ici : de par son exotisme, ses détours, ses escaliers dérobés, son architecture et ses jardins fantasques, le Village envoûte les spectateurs. Il y a bien manipulation mais elle n'est cette fois investie que de louables desseins : créer, par déplacement de perspective, des illusions dans le simple but de plaire. En suivant Numéro 6 dans sa découverte graduelle de ce décor fascinant, nous nous égarons avec délice sur les fausses pistes et fauxsemblants. Le Village transpose ainsi les spectateurs dans un espace ludique et artistique qu'ils ont plaisir à retrouver. Si l'épisode 16, " Once Upon a Time », est l'occasion pour Numéro 2 de rappeler avec Shakespeare l'artificialité de l'existence ( All the world is a stage »), le Village est une scène de choix en ce qu'il présente une double théâtralité. En effet, il est à la fois le décor de la série et le site réel mais

${ }^{10}$ Un exemple du fonctionnement du Rôdeur peut être visionné ici (lien consulté en août 2012) : http://www.youtube.com/watch?v=I6Ffr1U7KMY\&feature=related 
quelque peu factice du village-hôtel Portmeirion, dans la communauté de Penrhyndeudraeth, sur les côtes galloises ${ }^{11}$.

Ce vrai/faux village est une mise en scène de l'architecte gallois Sir Clough Williams-Ellis (1883-1978) élaborée entre 1925 et 1976. Sa variété architecturale est déconcertante et puise dans les styles espagnols, autrichiens, italiens, ou coloniaux. Des géographies, mais aussi des temporalités différentes, se superposent et s'entrelacent ${ }^{12}$. Le gothique, le baroque et le néo-classique y côtoient l'orientalisme dans une conception ludique mais toujours respectueuse des bâtiments. En effet, le parti pris de Portmeirion est de magnifier l'architecture, de lui offrir un écrin dans lequel l'artiste pourra gommer les défauts et imperfections inhérents à la réalité. L'image créée sera plus belle que le vrai, et placée sous le signe de la transposition: beaucoup de constructions ont, en effet, été récupérées de chantiers de démolition puis restaurées. D'autres sont en fait des trompe-l'œil : les élégantes fenêtres néoclassiques de la façade principale («La Piazza ») sont en réalité peintes et étirées pour donner davantage de prestance au bâtiment ; la fraîcheur végétale est une illusion (si les cyprès refusent de pousser, on les peint en vert) ; les proportions du Belvédère se rétrécissent considérablement vers le haut afin de le faire apparaître plus vertigineux. Un point de fuite est décalé, l'horizon est plus bas, et les figures surgissent, glorifiées par l'art de la perspective. L'artiste s'amuse à tricher et les visiteurs désirent être abusés, consciemment. Ils éprouvent la même sensation que lorsqu'ils assistent à une séance de prestidigitation. Par les différentes techniques de manipulation visuelle, Portmeirion semble nous mettre au défi : " Regardez bien et je vous prouverai que ce que vous pensiez impossible existe vraiment ! ». De ce point de vue, le choix de Portmeirion comme décor de la série ne peut être anodin. C'est un village-énigme qui instaure une dynamique ludique entre le créateur et les spectateurs. Et nous nous laissons volontiers emmener à l'écart dans ce labyrinthe enchanteur ${ }^{13}$.

Si le Village n'est pas un labyrinthe au sens «classique» du terme, les principes clés de complication de plan et de difficulté de déplacement sont bien présents. Il s'agit de circonscrire dans le plus petit espace possible l'enchevêtrement le plus complexe de sentiers, de

\footnotetext{
${ }^{11}$ Une carte de la situation géographique de Portmeirion (Pays de Galles) est consultable à l'adresse suivante : http://www.villagefederal.org/Portmeirion-Pays-de-Galles-le.html (lien consulté en août 2012)

12 Différents exemples d'architecture du village peuvent être vus aux adresses suivantes (liens consultés en août 2012) :

http://www.flickr.com/photos/retroweb/4306215663/

http://www.flickr.com/photos/retroweb/4301904596/in/photostream

http://www.gardenvisit.com/garden/portmeirion gardens

http://www.terresceltes.net/Portmeirion.html

${ }^{13}$ Une visite de Portmeirion, sur les traces du Prisonnier, peut être visionnée ici : http://www.youtube.com/watch?v=040RdQmyW5Y\&feature=related (lien consulté en août 2012).
} 
carrefours, de contournements afin de retarder l'arrivée du voyageur au centre qu'il veut atteindre. Dans Le Prisonnier, la caméra suit sans relâche les déplacements de Numéro 6 dans le Village. Ruelles, porches, escaliers, angles, carrefours, façades en trompe-l'œil foisonnent sur son parcours. Il semble passer et repasser sur les mêmes sentiers sans jamais trouver la combinaison libératrice, le bon enchaînement qui le mènerait hors champ. Il tourne en rond, prisonnier des méandres du Village. Cet espace devenu impossible fait écho à une esthétique en développement dans les années 60 : la fascination pour les labyrinthes (mythiques mais aussi modernes) et les dédales urbains. C'est par exemple le cas de l'exposition Dylaby - Un labyrinthe dynamique, en 1962 à Amsterdam sous l'impulsion de Jean Tinguely et Niki de SaintPhalle, ou de l'événement Une journée dans la rue, en 1966 à Paris, où les artistes du GRAV exploitent l'architecture et les méandres des rues comme un labyrinthe tout trouvé. En 1969, pour le film Rape, Yoko Ono choisit le mode de la filature et, en véritable Minos, lance un cameraman à la poursuite d'une inconnue dans les rues de Londres, jusque dans l'intimité de sa demeure. Les exemples seraient encore nombreux qui renoueraient avec la forme légendaire du labyrinthe ; tous présentent cette volonté de faire sortir les spectateurs et citadins de leur léthargie. Introduisant du jeu dans un système pétri d'automatismes et d'habitudes et pointant la participation active du corps dans son environnement, ces plasticiens remettent l'identité individuelle au centre d'une société parfois aliénante. Et l'on voit ici le rapprochement qui s'opère avec Le Prisonnier, cri d'alarme contre l'aliénation de l'individu par le détournement des technologies et de la science au service des pouvoirs. Le fil d'Ariane de Numéro 6, c'est son injonction :

I will not make any deal with you. I've resigned. I will not be pushed, filed, stamped, briefed, debriefed or numbered. My life is my own [...] I am not a number. I'm a person [...] I am a free man.

(Épisodes «Arrival », «Fall Out », générique. Cette réplique-leitmotiv agit comme un memento pour Numéro 6 qui ne doit pas perdre sa raison dans le labyrinthe surréaliste du Village).

Les différents Numéros 2 s'attacheront malgré tout à désorienter Numéro 6 et, en cela, le Village se révèle une arme redoutable, créant des univers parallèles (celui, par exemple, du western, dans l'épisode 14 " Living in Harmony ») qui s'interpénètrent et s'échangent. Ce thème est, dans les années 60, la préoccupation principale de l'artiste M.C. Escher (1898-1972), adepte des figures impossibles, de l'étude du mouvement perpétuel et des figures labyrinthiques. Par des jeux troublants de perspective, il parvient à faire coïncider des mondes distincts, où des créatures peuvent être en plusieurs endroits au même moment, à la fois descendre et monter des escaliers. Alors qu'il nous fait perdre nos repères sensoriels, il 
démontre qu'une réalité est toujours double, qu'un objet peut être convexe et concave à la fois, plein et creux.

Sa lithographie Montée et Descente (1960) ${ }^{14}$ montre un château en plongée, qui parait normal au premier coup d'œil. En y regardant de plus près, on remarque que des personnages descendent un escalier situé au sommet d'un atrium. Curieusement, ils croisent d'autres personnages qui montent ce même escalier... sans fin. En effet, si nous suivons la file ascendante, on constate qu'elle ne redescend jamais, et que l'escalier se boucle sur lui-même en ne faisant que monter (ou descendre si l'on choisit de suivre la file descendante). Les personnages vont passer et se croiser sans cesse pour revenir toujours au point de départ. Certains passages du Prisonnier apparaissent comme une transposition de cette simultanéité impossible. Dans l'épisode «Arrival », par exemple, Numéro 6 reçoit la visite d'un réparateur électrique. Ce dernier a le crâne rasé et porte une combinaison couleur chair. Après un bref échange, Numéro 6 sort de chez lui pour « faire un tour », ou plutôt pour « tourner en rond », mais il ne le sait pas encore. Il monte des escaliers et tombe, déconcerté, sur le réparateur qu'il vient pourtant de quitter (voir figure 3).

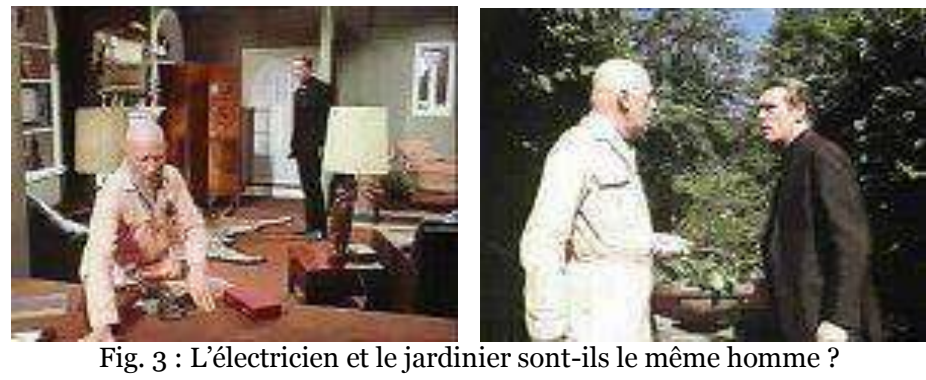

Il s'agit bien du même acteur chauve en combinaison chair mais il est pourtant logiquement impossible que ce soit le même personnage qui descende les marches à ce moment-là. Est-ce une illusion ? Numéro 6 est-il prisonnier d'un escalier de Penrose, cette structure utilisée par Escher dans Montée et Descente? Pouvons-nous toujours faire confiance à nos sens et à notre cerveau pour interpréter nos perceptions ? Un peu plus tôt, Numéro 2 avait prévenu Numéro 6 de son penchant pour les gadgets futuristes et les jeux d'optique : " Aha, I'm sorry, I can never resist that ». Il semble qu'en effet on nous joue des tours dans le Village et, de ce point de vue, le nom du pub («The Cat and Mouse », dans l'épisode 2 « The Chimes of Big Ben ») n'est

${ }^{14}$ Maurits Cornelis Escher, Montée et Descente, lithographie, 1960, 35x28,5 cm. Elle peut être vue à l'adresse suivante: http://fr.denhaag.nl/fr/residents/to/Musee-Escher-aupalais.htm (lien consulté en août 2012). 
certainement pas anodin. Qui est le chat et qui est la souris ? Et surtout, qui tire les ficelles - qui est Numéro 1 ? Le prisonnier essaiera de le découvrir tout au long des épisodes : "I intend to discover who are the prisoners and who are the wardens » (épisode 4 «Free for All »). Mais le Village ne livre pas ses secrets, il nous fait tourner en rond. Il ne se livre pas à une lecture qui trouverait dans l'image de quoi articuler une logique ou une intrigue claire. C'est un piège pour Numéro 6 mais c'est aussi un "piège à spectateurs ».

Le Village est déstabilisant parce qu'il nous amène progressivement à une conception du lieu et des figures qui n'a plus rien à voir avec l'espace organisé et rationnel de la perspective géométrique, mais plutôt avec les espaces intimes de l'imagination et de l'onirisme. Cette transposition est à l'origine d'un déploiement surréaliste de motifs et d'intrigues carnavalesques et incongrus, conférant à la série une poétique à part entière. Parce qu'elle nous incite à abattre les cloisons hermétiques entre notre conscience et les couches instinctives plus profondes de la psyché, cette poétique participe d'un mouvement plus large de décloisonnement de l'espace.

Dans son ouvrage L'Euvre de François Rabelais et la culture populaire au Moyen Âge et sous la Renaissance ${ }^{15}$, Mikhaïl Bakhtine décrit le carnavalesque comme un renversement temporaire des hiérarchies et des valeurs. Au Moyen Âge, le Carnaval était une puissante expression subversive. C'était l'occasion pour le peuple d'abattre, de façon symbolique et pendant une période limitée, les cloisons entre dominants et dominés, entre haut et bas, noble et trivial, sacré et profane. Ce renversement des valeurs culminait dans l'élection d'un roi occupant symboliquement et temporairement les espaces du pouvoir. Plusieurs épisodes transposent cette définition de Bakhtine à l'univers du Prisonnier et participent d'une stratégie sous-jacente de décloisonnement. Dans l'épisode 4 "Free for All », Numéro 6 se présente à une élection pour tenter de supplanter Numéro 2. Il s'agit bien évidemment d'une mascarade qui exploite le motif du carnavalesque. De nombreuses parades colorées sont organisées dans l'enceinte du Village par "the madding crowd », pour reprendre les termes de Numéro 2, et tous les objets scéniques du Carnaval sont là : ballons, parapluies et costumes bigarrés, klaxons, fanfare et confettis. Mais de renversement, il n'y aura vraiment que celui du nouveau roi, Numéro 6. Renversement politique, puisqu'il a gagné les élections mais sera immédiatement destitué ; mais aussi renversement spatial : lors d'un interrogatoire éprouvant, Numéro 6 est soumis à l'épreuve du vertige (son pupitre l'emporte dans une ronde folle qui lui fait perdre équilibre et conscience ; la caméra subjective nous fait adopter sa

${ }_{15}$ Mikhaïl Bakhtine, L'Euvre de François Rabelais et la culture populaire au Moyen Âge et sous la Renaissance, Paris, Gallimard, Coll. Tel., 1965. 
perspective et nous avons le sentiment, nous aussi, de tomber à la renverse). Cette première tentative de décloisonnement est relayée par le discours, notamment à l'aide du zeugme, cette figure de style qui rapproche deux termes habituellement disparates, brouillant ainsi les cloisons lexicales, et de l'anacoluthe (les échanges, incompréhensibles, avec la chargée de campagne électorale développent une syntaxe de la rupture). L'épisode 8 , « Dance of the Dead ", reprend le thème du Carnaval :

All citizens take note that Carnival is decreed for tonight. Turn back the clock. There will be music, dancing, happiness, all at the Carnival. By order.

Les aiguilles du temps sont inversées mais il semble que les valeurs basculent également. Lors d'un bal costumé, les résidents sont invités à mal se comporter: «If the people misbehave, we'll give them a 58 » (du whisky comme récompense). Le haut et le bas sont inversés : le petit peuple incarne Élisabeth Ire, Jules César, Cléopâtre ou encore Napoléon. Les repères identitaires sont remis en question à travers le motif du masque des résidents mais aussi et surtout à travers le nondéguisement de Numéro 6. Lorsqu'une serveuse lui demande pourquoi il n'a pas de déguisement, Numéro 6 explique qu'il a, en fait, conservé son identité, contrairement aux autres habitants (SERVEUSE. "What does that mean ? "/ NumÉRo 6. "That I'm still myself. »). Mais face à la réponse de Numéro 2 à la même question, il est forcé de reconnaître la possibilité d'un flou identitaire (Numéro 6. "Why haven't I [got] a costume? »/ NumÉRo 2. "Perhaps because you don't exist. »). Nous basculons alors dans un monde absurde, symbolisé par un simulacre de procès aux accents kafkaïens au terme duquel Numéro 6 sera condamné à mort. L'épisode se conclut en queue de poisson sur une course poursuite désordonnée et le rire sardonique de Numéro 2, exceptionnellement représenté par une femme déguisée en Peter Pan. Les intrigues suivantes exploiteront de plus en plus les situations étranges, proches du rêve - ou du cauchemar - démantelant progressivement les cloisons de la logique.

En tentant de transposer le monde du rêve et du délire dans la réalité, en laissant libre cours à une imagination de plus en plus débridée, la série se rapproche ostensiblement de l'esthétique surréaliste. En effet, si être surréaliste, c'est poser en permanence plus de questions qu'on en résout ${ }^{16}$, Le Prisonnier participe sans conteste de cette dynamique. Au cœur du surréalisme, il y a une énigme qui doit rester telle pour les spectateurs. La raison pour laquelle Le Prisonnier est une série déroutante, peut-être même décevante pour certains, c'est qu'aucune explication rationnelle n'est apportée à l'énigme qu'elle

${ }^{16}$ Dominique Bouquet, Le Surréalisme en France et en Europe, Paris, Les Guides Pocket Classiques, 2003, p. 8. 
constitue. Dans une interview pour la télévision conduite par Mike Smith dans les années 70, Patrick McGoohan déclara :

The reason that it was confusing, and that [the viewers] were disappointed, I think, was that they expected the ending to be similar to a "Bond" thing, with this mystery man, the head man or whatever they call him in Bond; and of course it wasn't about that at all. It was about the most evil human "being", human "essence"; and that is ourselves, because within each of us, that is the most dangerous thing on Earth, is what is within us. And so therefore that's what I made No. 1: One's "self", an image of himself that he was trying to beat ${ }^{17}$.

Dans le Premier Manifeste du Surréalisme ${ }^{18}$, André Breton met en avant la dimension inconsciente du mouvement : les artistes doivent se faire l'écho des strates sous-jacentes de la pensée pour donner à voir ce que McGoohan décrit comme dangereux : notre propre espace intime, ou théâtre intérieur. Nous sommes conscients de l'étrange mais nous suivons le cheminement de l'artiste en éprouvant la sensation déstabilisante d'une mise hors circuit. On pense, dans le contexte des années 60, à la découverte des psychotropes hallucinogènes ouvrant les " portes de la perception ${ }^{19}$ », mais pas seulement. Au gré des situations loufoques et parfois incompréhensibles que nous proposent les derniers épisodes (citons en particulier l'épisode 15 «The Girl Who Was Death », l'épisode 16 " Once Upon a Time » et l'épisode 17 "Fall Out »), nous quittons définitivement l'univers objectif et rationnel.

Selon C. G. Jung, la singularité de notre existence vient de la certitude qu'il existe une psyché inconsciente, deux personnalités dans le même individu, ou, pour reprendre ses termes, un Moi et son " ombre ${ }^{20}$ »; or, nous vivons dans un espace trop objectif, dépouillé de toute "participation mystique ${ }^{21}$ » et il est impératif de se reconnecter avec le monde primitif de l'instinct sous peine de voir notre psyché se fragmenter. C'est à ce centre identitaire que nous convie le labyrinthe psychédélique du Prisonnier ; il nous invite à remonter le fil d'Ariane, à retrouver le chaînon manquant (" [we must] find the missing link », épisode 16 "Once Upon a Time »). Ce cheminement ne peut se faire que par le rêve, lieu privilégié des décloisonnements de la pensée (et thème central de l'épisode 3 « $\mathrm{A}, \mathrm{B}$ and $\mathrm{C} »)$. En effet, les images et les idées se conservent, à l'état subliminal, à un niveau de tension très

${ }^{17}$ Cet entretien peut être visionné ici : http://www.youtube.com/watch?v=RhlS6kNTopI (lien consulté en août 2012).

${ }^{18}$ André Breton, Les Manifestes du surréalisme, Paris, Pauvert, Gallimard, coll. Idées, 1962. 19 « The Doors of Perception » est le titre d'un essai d'Aldous Huxley, paru en 1954, dans lequel l'auteur relate sa première expérience de la mescaline, une substance psychédélique. Ce titre est emprunté à William Blake : "If the doors of perception were cleansed every thing would appear to man as it is, infinite », William Blake, The Marriage of Heaven and Hell, plate 14, «A Memorable Fancy » (1790-1794).

${ }^{20}$ Carl Gustav Jung, Essais d'exploration de l'inconscient, Paris, Éditions Robert Laffont, Folio Essais, 1964, p. 145.

${ }^{21}$ Jung, p. 69. 
inférieur à celui qu'elles ont dans la conscience. Dans cet état, elles perdent la clarté de leur contour et se fondent les unes aux autres. Une histoire racontée par notre esprit conscient a un début, un développement et une conclusion facilement restituables car bien cloisonnés. Il n'en va pas de même du rêve ; et il n'en va pas de même non plus du Prisonnier. La méthode d'analyse des rêves conçue par Jung nous amène plutôt à effectuer une sorte de «promenade circulaire qui aurait l'image du rêve pour centre ${ }^{22}$ ». À bien y regarder, les mouvements de caméra incitent à cette promenade circulaire.

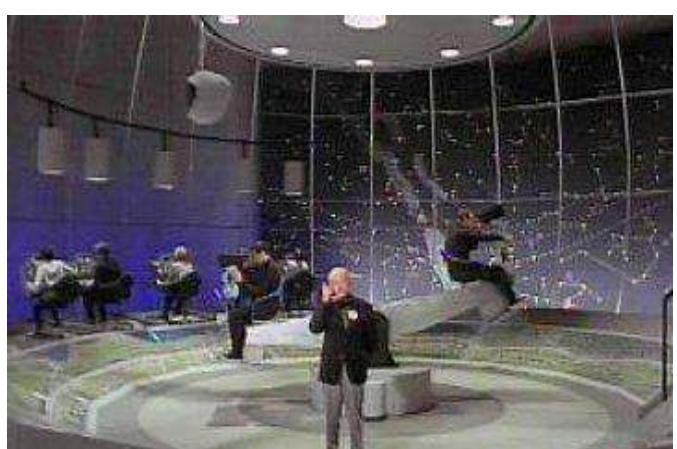

Fig. 4 : Épisode 1 « Arrival ». Un des nombreux motifs circulaires de la série : au centre du dôme, cette station d'observation possède un siège de commande pivotant autour de l'axe central. Le motif circulaire est répété plusieurs fois au sol et au plafond.

Qu'ils suivent le vol d'une mouette, la course de Numéro 6 sur la plage, qu'ils survolent le Village ou les différentes salles de contrôle - toutes circulaires, voir figure 4 - ces mouvements imposent aux spectateurs une vision tourbillonnante, comme pour donner le vertige, nous faire rentrer dans un état semi-hypnotique, proche de l'état du rêve. Il nous reste alors à pénétrer cet espace onirique.

L'épisode le plus représentatif, celui qui nous fait incontestablement et irrémédiablement tomber dans cet espace décloisonné, c'est le tout dernier, «Fall Out ». Situé symboliquement au plus profond d'une caverne secrète, il transpose et condense, tel un rêve, une multitude d'images et de situations déjà connues des spectateurs : les Numéros 2 de « The Chimes of Big Ben » (épisode 2), de "Once Upon a Time» (épisode 16) et de "The Girl Who Was Death » (épisode 15), le jeune aliéné de « Living in Harmony » (épisode 14), le motif du masque - les acteurs portent un masque de tragédie grecque noir et blanc -, le trône du Carnaval, le balancier de la salle de contrôle, le procès kafkaïen, la course poursuite, des chercheurs dans

${ }^{22}$ Jung, p. 46. 
un laboratoire, la fusée de « The Girl Who Was Death »... La musique, dont on ne sait pas vraiment si elle appartient à la diégèse ou pas, est en totale contradiction avec l'atmosphère oppressante de l'épisode : des passages de All You Need is Love des Beatles scandent une fusillade, le Negro Spiritual Dem Bones est utilisé comme plaidoirie, des alarmes se mélangent à For He's a Jolly Good Fellow. Comme dans un rêve, des images et des sons qui semblent contradictoires et parfois incongrus sont associés, mélangés, échangés. Les choses les plus banales peuvent revêtir un aspect enchanteur ou effrayant par le jeu des déplacements et des transferts. Au terme de son séjour au Village (au cours duquel il aura subi toutes les techniques de manipulation psychologique et d'exploration de l'inconscient), le héros n'est plus un numéro et a retrouvé l'honneur d'être un homme : " the right of an individual to be an individual ${ }^{23} »$. Le dénouement est proche, la confrontation tant attendue avec le Numéro 1 va enfin lever le voile sur nos interrogations. Qui est-il24? Une figure cagoulée et masquée de noir et de blanc - le ying et le yang, une personnalité double. Numéro 6 arrache le masque et révèle... un grand singe (voir figure 5). L'homme est un animal. Mais il y a une autre épaisseur, un autre masque à ôter. Nous découvrons avec stupeur un visage humain, celui, grimaçant, du Prisonnier! (voir figure 6)
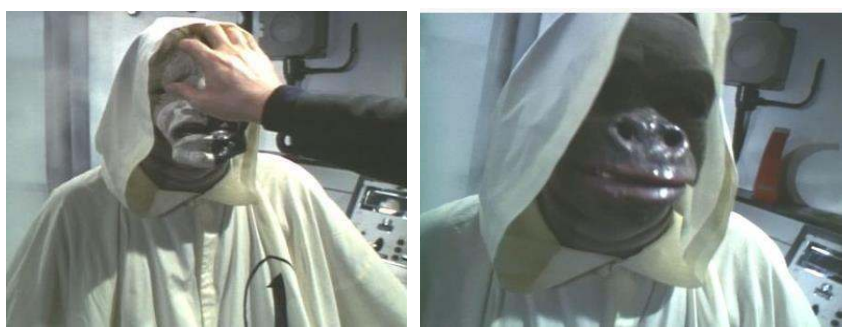

Fig. 5 : les différentes strates de la personnalité de Numéro 1

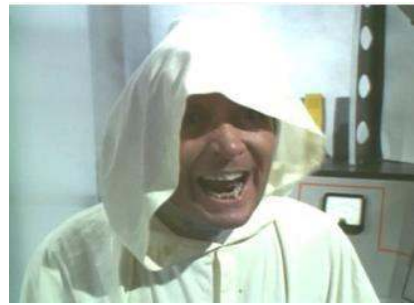

Fig. 6 : Le coup de théâtre

${ }^{23}$ Phrase prononcée par le juge lors de la plaidoirie du dernier épisode.

24 Cette scène (commentée) peut être visionnée ici (lien consulté en août 2012) : http://www.youtube.com/watch?v=X114df65hgY 
L'homme est son propre geôlier. Il ne pourra réellement se libérer que s'il accepte de se confronter à son "ombre " et à l'accepter. Les félicitations du juge-Numéro 2 prennent alors tout leur sens: "We applaud his [Number 6's] private war ». L'espace intime est devenu l'enjeu d'une réalité toujours double (on repense ici à Escher). Bien sûr, le spectateur attentif aura repéré des indices de ce final - écrit dans la précipitation, puisque les producteurs avaient décidé de mettre fin prématurément à la série. L'adresse londonienne du prisonnier était prémonitoire (il habite au numéro 1), l'intonation, volontairement ralentie, du Numéro 2 dans le générique pouvait jeter le trouble (NUMÉRO 6. "Who's Number 1?» / NUMÉRO 2. "... You... are... Number 6 ", qui peut être interprété comme "Vous, vous êtes le Numéro 6 » ou bien «C'est vous, Numéro 6 »). De plus, le thème du doppelgänger avait déjà été traité dans les épisodes 5 "The Schizoid Man » et 13 « Do not Forsake Me, Oh My Darling ».

Que devons-nous conclure de tout cela? À nous poser la question, nous renouons certainement avec ce que McGoohan a voulu dénoncer: la volonté excessive de tout contrôler et rationnaliser. Reconnaissons-le, une grande partie du charme et de la pérennité du Prisonnier réside dans cette incertitude, ce décloisonnement total de nos repères rationnels. Le Village reste indécodable; on ne peut que le surcoder des différentes strates issues de notre imagination, de notre propre vie onirique.

Au terme de la série, le Village n'a donc pas livré tous ses secrets. Creuset d'une esthétique dystopique et carcérale, il participe incontestablement à la dimension prophétique du Prisonnier, cri d'alarme contre les dangers d'une société qui chercherait à anéantir tout espace individuel. Mais le Village est aussi un lieu de séduction qui entretient un jeu intime avec le spectateur en troublant sa perspective. En un mot, nous sommes captivés autant que Numéro 6 est captif. Dans son œuvre subversive, McGoohan crée un univers qui nous invite à déplacer plus fondamentalement encore nos perspectives en explorant des territoires mystérieux, ceux de l'intime et de l'inconscient. En mettant en scène son théâtre intérieur, il suggère que l'homme n'atteint réellement sa complétude que s'il accepte d'abattre les cloisons et de se connaître vraiment. Dans ce territoire inconnu que sont les tréfonds de son âme, l'homme doit accepter ce qu'il a de plus mystérieux et déstabilisant (Numéro 6 est confronté tout au long des épisodes à ses penchants impulsifs, colériques, ou même paranoïaques) pour devenir maître de soi et de son destin. Il faut aussi prendre conscience des conditionnements inhérents à toute société («We're all pawns ", s'écrie l'Amiral dans le premier épisode). La toute dernière image de la série nous ramène à la perspective initiale : la route déserte, le point de fuite infini. La promenade circulaire est bouclée. Mais dans 
cette espace dorénavant ouvert, le héros est sûr de sa destination : au volant de sa Lotus Seven, son visage est déterminé, il est désormais maître de sa destinée.

\section{Bibliographie}

BAKHTINE Mikhaïl, L'Euvre de François Rabelais et la culture populaire au Moyen Âge et sous la Renaissance, Paris, Gallimard, Coll. Tel., 1965.

BOUQUET Dominique, Le Surréalisme en France et en Europe, Paris, Les Guides Pocket Classiques, 2003.

BRETON André, Les Manifestes du surréalisme, Paris, Pauvert, Gallimard, coll. Idées, 1962.

FouCAUlT Michel, Surveiller et Punir, Paris, Gallimard, Coll. Tel., 1975 .

HAWTHORNE Nathaniel, The Scarlet Letter, New York, Penguin Books, 2003 [1850].

JUNG Carl Gustav, Essais d'exploration de l'inconscient, Paris, Éditions Robert Laffont, Folio Essais, 1964. 